\title{
II.
}

\section{Die naturphilosophischen Ideen bei Cyrano de Bergerac.}

\author{
Von
}

\section{A. W. Loewenstein.}

\section{Einleitende Bemerkungen ( $\S 1-4)$.}

\section{$\S 1$. Gegenstand.}

Es ist bemerkenswert und lehrreich, zu sehen, wie die gewaltigen Geistesströmungen, die durch die Jahrhunderte gehen, und deren Quelle und Verlauf die geschichtliche Forschung an große Namen knüpft, sich widerspiegeln in den Köpfen kleinerer Geister. Nie dasselbe Gepräge tragend und doch der Richtung des großen Stromes folgend, geben die wechselnden Ideen dieser mehr reproduzierenden als produzierenden Männer dem Gesamtbild eine um so anziehendere Mannigfaltigkeit. Nicht jeder von ihnen kann einen eigenen Platz in dem Rahmen der ganzen Geschichte beanspruchen; aber oft dient die Art und Weise, wie die kleineren Genies die Ideen eines grösseren auffassen, zu einer nützlichen Illustration gerade der letzteren selbst. Aus der grossen Schar der Planeten und Trabanten, die um die aufgehende Sonne der neueren Philosophie zu Anfang des 17. Jahrhunderts kreisen, wollen wir einen auswählen, dessen Name unter den Philosophen bis jetzt nicht genannt wurde. Savinien de Cyrano Bergerac ist allerdings in unseren Tagen berühmt geworden. Über die Bühnen 
des Abendlandes ging ein Heldendrama, ${ }^{1}$ ) das hervorgebracht zu haben, sich die Franzosen als Verdienst anrechnen können. Aber die historische Persönlichkeit ließ es im Dunkeln. P. A. Brun ${ }^{2}$ ) ist wohl der Einzige, der sich ernsthaft um Cyrano bekümmert hat. Doch seine Arbeit ist eine literarhistorische. In einigen Kapiteln behandelt er allerdings auch seine wissenschaftlich-philosophischen Ideen, indes in wenig genügender Weise. Bei Gelegenheit soll darauf hingewiesen werden.

\section{$\S 2$. Cyranos äußeres Leben.}

Dank den eifrigen und erfolgreichen Bemühungen des Bibliophilen $\mathrm{Jal}_{,}{ }^{3}$ ) wissen wir, daß Cyrano am 6. März 1619 in der Pfarrei Saint-Sauveur zu Paris, als der Sohn einer adligen Familie, geboren wurde. Zuerst bei einem Priester auf dem Lande, dann im Collège de Beauvais (seit 1631) erzogen, zeigte er schon von Jugend an einen wilden, zügellosen Charakter. Die Notizen über sein Leben verdanken wir seinem Freund Lebret. ${ }^{4}$ ) Nach Beendigung seiner Studien (1637) wusste er seine Zeit nicht besser anzuwenden, als sich einem zügellosen, ausschweifenden Leben hinzugeben. 1638 veranlaßte ihn Lebret, der in dieser Ausnutzung der Freiheit nur Unheil für seinen Freund kommen sah, in die Garden als Soldat einzutreten, und zwar in das von dem Kapitän Carbon de Castel-Jaloux kommandierte Regiment der cadets de Gascogne. Das Soldatenleben war für diesen unbändigen Geist wie geschaffen. Bald nannten ihn seine Kameraden nur noch den démon de la bravoure. Überall hatte er Ehrenhändel, infolge der geringfügigsten Anlässe. Fast kein Tag verging, an dem er nicht ein Duell gehabt, oder als Sekundant gedient hätte. 1639 kämpfte er gegen die Deutschen bei Mouzon und erhielt einen Musketenschuß. 1640 lag er vor Arras und bekam einen Säbelhieb am Hals. Mancherlei Gründe: seine Verwundungen, die Neigung zur

1) Edmond Rostand, Cyrano de Bergerac. Comédie héroique; Paris 1897.

2) P. A. Brun, Savinien de Cyrano Bergerac. Dissertation. Paris 1894

3) Jal, Dictionnaire biographique.

4) Lebrets Préface zu den „Oeurres" des Cyrano. 
Wissenschaft, die in ihm erwachte, vor allem aber die Liebe zur Unabhängigkeit und Freiheit veranlaßten ihn, das Kriegshandwerk aufzugeben. Jetzt erst beginnt ernsteres Studium und tiefere Beschäftigung mit den Wissenschaften. Gassendi eröffnete gerade damals eine Privatvorlesung. Man wollte Cyrano nicht zulassen. Doch er erzwang sich die Erlaubnis durch Drohungen. Und so wurde er Gassendis Schüler zusammen mit Chapelle, Molière, Bernier, Hesnaut und anderen. Aus dieser Zeit, in der auch Campanella und Michel de Marolles zu seinen Freunden rechneten, stammen die uns von ihm erhaltenen Werke. ${ }^{5}$ ) Sein Leben indes bleibt auch fernerhin charakterisiert durch seine Unbändigkeit. Die beiden Episoden, die Rostands erster Akt schildert, sind von Lebret als wahr überliefert. Sein gerader, aufrechter Charakter mußte überall anstoßen. Seine Unabhängigkeit und sein Freiheitsdrang sträubten sich dagegen einen Gönner zu wählen, wie des klassischen französischen Jahrhunderts Brauch war. Er wollte allein stehen und kämpfen. So kam es, daß er der Freunde nur wenige, dagegen viele der Feinde zählte. Jung, wahrscheinlich in bedrängten, elenden Verhältnissen, ist er gestorben, im September 1655, 36 Jahre alt. Ein Balken, vielleicht von feindlicher Hand geschleudert, verletzte ihn tödlich.

\section{§ 3. Hauptzug seines Charakters.}

Dies in kurzen Zügen sein Leben, in dem sich seine amour farouche de l'indépendance als wichtige Seite seines Charakters schon scharf abhebt. Und wie er in seinem Leben von niemandem abhängig sein wollte, so auch in seinen Ideen. „Die Vernunft allein ist meine Königin“, ruft er aus, „vor niemandes Autorität beuge ich mich, sie sei denn durch Vernunft gestützt.“6) Er

5) Les œuvres diverses de Monsieur Cyrano de Bergerac, en 3 vols Amsterdam, chez Desbordes. 1741. 1 vol: Histoire comique des Etats et Empires de la Lune et du Soleil. 2 vol: Les Lettres; Fragment de Physique. 3 vol: Le pédant joué, comédie; la mort d'Agrippine, tragédie.

6) Cyrano II, 82. Lettre contre les sorciers: Je ne défère à l'autorité de personne, si elle n'est accompagnée de raison, ou si elle ne vient de Dieu, Dieu qui tout seul doit être cru de ce qu'il dit à cause qu'il le dit. 
machte sich lustig über Leute, die mit einem Satz des Aristoteles etwas beweisen wollten, oder die, wie die Pythagoräer, mit einem „Magister dixit" den Gegner aus dem Felde schlagen zu können glaubten. Lebret berichtet, nächst Sokrates seien ihm Demokritos und Pyrrhon am vernünftigsten erschienen, der erstere wegen seiner Unklarheit, der zweite, weil er so bescheiden sei, niemals etwas entscheiden zu wollen. ${ }^{7}$ ) Nutzlose philosophische Fragen verachtete er, und Gefallen bereitete es ihm zu lesen, daß Theophilus den Sidias in seinen komischen Fragmenten verprügeln läßt, weil derselbe sich auf der Behauptung versteife: odor in pomo non est forma sed accidens. ${ }^{8}$ ) Den Philosophen seiner Zeit wirft er vor, sie seien Kompilatoren und Abschreiber, ja schlimmer als Diebe und Straßenräuber, da sie ihren Ruhm nur dem, was sie von anderen gestohlen hätten, verdankten. $\left.{ }^{9}\right)$ Wir gehen wohl nicht fehl, wenn wir hierin einen Hieb vor allem gegen Gassendi sehen, der ja allerdings Epikur und Lukretius sein System und seine Ideen entleiht, aber gewiß obigen Vorwurf am allerwenigsten verdient. Auch die Kritiker sind Cyrano eine unausstehliche Nation, denn ihre Tadelsucht stamme doch nur her aus dem Bewußtsein ihrer eignen Unfähigkeit. ${ }^{10}$ )

\section{$\S 4$. Cyranos Abhängigkeit von seiner Zeit.}

Obgleich also, wie wir sahen, Cyrano auf sein Wappenschild schrieb: Nullius addictus jurare in verba magistri, so würden wir doch fehlgehen, wenn wir annähmen, er gehe nun ganz seinen eigenen Weg für sich. Der Mensch ist le produit de la race, du moment, et du milieu, sagt Taine in etwas übertriebener $\mathrm{Zu}$ schärfung einer an sich richtigen Idee. So konnte sich auch Cyrano dem großen Strudel der Gedanken seines Jahrhunderts nicht ent-

Ni le nom d'Aristote, plus savant que moi, ni celui de Platon, ni celui de Socrate ne me persuadent point, si mon jugement n'est convaincu par raison de ce qu'ils disent: La raison seule est ma reine.

7) Cyr. I, XIV Préface.

8) Cyr. I, IX Préface.

9) Cyr. I, XV Préface.

10) Cyr. I, XVI Préface. 
ziehen. Allerdings ist zuerst ein Bedenken zu beseitigen, das uns vielleicht zwingen könnte, ihm doch konsequentes Festhalten an seinem Motto zuzuerkennen. Das Werk nämlich, das wir vor allem unserer Kenntnis von Cyranos Ideen zu Grunde legen müssen, Le voyage comique à la lune et au soleil, trägt einen komischen und satirischen Charakter. Haben wir also das Recht, irgend einen der dort ausgesprochenen Gedanken als ernst anzunehmen? Müssen wir nicht vielmehr vermuten, daß Cyrano, wie in manchen Dingen ganz offenbar, so auch im ganzen nur amüsiert, sich lustig macht und den Leser zum Narren hält? Doch diese Gefahr ist beseitigt dadurch, daß wir ein Fragment ernsten Charakters haben, das Fragment de Physique, das uns so in etwa als Maßstab dienen kann. Es ist aber gleichwohl zu bemerken, daß es darauf ankommt, Cyranos ernste Gedanken oder deren Ursprung aus dem Rahmen und der Hülle herauszuschälen, in die ihn die blühende Phantasie unseres Autors so oft kleidet. Denn Cyrano ist vor allem auch Poet; und als solcher lebte er unter dem Einflusse des Burleskenund Preziösentums.

Ebenso wie in Bezug auf die Form, war er auch in der Hauptsache seiner Ideen ein Sohn seiner Zeit. Gerade sein Sträuben gegen das Anerkennen der Autorität alter Philosophen kennzeichnen ihn als solchen. Den Priester, bei dem er auf dem Lande zuerst erzogen wurde, nennt er schon als Knabe ${ }^{11}$ ) „un âne aristotélique“; und gerade gegen Aristoteles richtete sich ein großer Teil der damaligen philosophischen Streitliteratur. ${ }^{12}$ ) Der Gegensatz gegen aristotelische Ideen ist ein wichtiger Punkt in dem Programm der Philosophie des 16. und 17. Jahrhunderts. Aber das ist es nicht allein, was Cyrano mit seiner Zeit gemeinsam hat. Es wäre wenig.

Die Voraussetzungen philosophischen Denkens bei Cyrano. $(\S \S 5-7)$.

$\S 5$. Descartes' methodischer Zweifel.

Es ist für uns zunächst wichtig, festzustellen, was für Cyrano Voraussetzung und Ausgangspunkt philosophischen Denkens war.

11) Cyr. I, Préface.

12) Bouillier, Histoire de la philosophie cartésienne. 2 vols. Paris 1854. I, 4 und 5 . 
Die beiden einleitenden Kapitel des Fragment de Physique ${ }^{13}$ ) klären uns darüber auf. Da sehen wir, wie gewaltig die Wirkung der neuen klaren Gedanken Descartes' ${ }^{\prime 4}$ ) gewesen sein muß. Denn wir finden bei dem Schüler Gassendis im Grunde nichts anderes als eine Wiedergabe von Hauptgedanken Descartes.. ${ }^{15}$ ) Der Ausgangspunkt des Descartes'schen Denkens ist der methodische Zweifel, der zur Wahrheit führen soll. ${ }^{16}$ ) Und so ist es auch bei Cyrano: Wir besitzen keine andere Kenntnis der uns umgebenden Gegenstände, als die durch unsre Sinneswahrnehmungen vermittelte. Und unsere Sinne sind trügerisch. ${ }^{17}$ ) Wenn wir einen Gegenstand außerhalb von uns sehen, so existiert er deswegen nicht wirklich. Im Traume haben wir ja auch Gesichtswahrnehmungen von Dingen, deren Existenz sicher nur im Geiste sein kann. ${ }^{18}$ ) Zahlreiche Beweise führt Cyrano für die Trüglichkeit der Sinne an, besonders aus dem Gebiete der Optik: ein im Kreise bewegter Kienspan erscheint dem Auge als feuriger Kreis. ${ }^{19}$ ) Das uns vom Spiegel gezeigte Bild kann sicher doch nur in uns existieren. ${ }^{20}$ ) Eine angezündete Kerze, mit zusammengekniffenen Augen betrachtet, sendet scheinbar nach oben und unten Strablen aus, die doch in Wirklichkeit nicht da sind; denn wenn man mit der Hand den unteren Teil der Kerze verdeckt, so erscheinen die unteren Strahlen, bedeutend genähert, auf der Hand; verdeckt man das ganze Licht, so verschwinden sofort obere und untere Strahlen. ${ }^{21}$ )

Es schwindet uns also der Boden unter den Füßen; denn die einzige Quelle unsres Erkennens ist trügerisch. ${ }^{\text {22) }}$ Hätte also unser

13) Cyr. II, 355-370.

14) Desc. zitiert nach V. Cousins Ausgabe in 11 Bänden. Paris 1824.

15) Desc. Médit. 1 (I, 235-245).

$\left.{ }^{16}\right)$ Desc. Méth. 3 (I, 153): Non que j'imitasse pour cela les sceptiques, qui ne doutent que pour douter, et affectent d'être toujours irrésolus; car, au contraire tout mon dessein ne tendait qu'à m'assurer.

17) Cyr. Il, 365.

18) Cyr. II, 360.

19) Cyr. II, 361.

20) Cyr. II, 362.

${ }^{21)}$ Cyr. II, 363.

${ }^{22)}$ Cyr. II, 364, $36 j$. 
Mißtrauen etwas Ungerechtfertigtes, wenn wir zu der Überzeugung kämen, unser ganzes Leben sei nur ein Traum? ${ }^{23}$ )

Bis hierher konstatieren wir ein fast genaues Zusammengehen mit dem Gedankengang Descartes' in den Meditationen I. Aber dann bricht Cyrano ab. Was dem Denken Descartes' den Hauptstempel aufdrïckt, die Sicherheit, mit der ihm aus dem Zweifel an allem die Existenz des Ich hervorgeht, ${ }^{24}$ ) und allmählich alles, was wir als wahr erkennen können, sicherer und fester fixiert wird, - von dem allem finden wir bei Cyrano nichts. Dagegen tut er einen Schritt weiter und erklärt die Sinne nicht nur für trügerisch, sondern auch für unzulänglich, um alle Dinge zu erklären. Hierin müssen wir eine Anlehnung an Gassendi sehen, ${ }^{25}$ ) der ebenfalls die Unvollkommenheit der Sinne betont. ${ }^{26}$ ) Der Dämon des Sokrates erklärt dem Cyrano auf dem Mond: Zu wenig Beziehung besteht $\mathbf{z w i s c h e n}$ deinen Sinnen und der Erklärung aller Wunder. Im Universum ' gibt es Millionen von Dingen, für deren Erkenntnis du Millionen verschiedener Organe nötig hättest. Ich erkenne Dinge, die für dich nur Gegenstand des Glaubens sein können. ${ }^{27}$ )

\section{$\S 6$. Die Existenz der Materie.}

Wir sehen, wie Cyrano plötzlich den Gedankengang Descartes' abbrach. Nichtsdestoweniger ist aber auch für ihn die Existenz der Materie Voraussetzung.

Nach den Descartes nachgeschriebenen Gründen für den Zweifel, fährt er nach einigen allgemeinen Bemerkungen fort, über die Materie zu sprechen, ${ }^{28}$ ) ohne auch nur ein Wort über den Weg zu verlieren, der Descartes zu deren Annahme führte. Wir können uns also kaum der Annahme verschließen, daß die eigentliche Bedeutung des methodischen Zweifels, wie ihn Descartes ausspricht, Cyrano verborgen geblieben ist; denn ohne die Unmöglichkeit

23) Cyr. II, 366: nous pourrions entrer en défiance que notre vie serait un songe continuel. Vergl. Desc. Médit. 1.

24) Desc. Médit. 2 (I, 247 f.).

${ }^{25)}$ Gassendi zitiert nach: Petri Gassendi opera, 6 vols Lugduni $1658.4^{0}$.

26) Gass. I, 268.

27) Cyr. I.

28) Cyr. II, 371.

Archiv f. Geschichte d. Philosophie. XVr. 1 
seines Unterfangens zu verstehen, schreitet er über den Abgrund leichten Fußes hinweg, der zwischen dem $Z$ weifel an allem und der Anerkennung der Körper als existierend liegt, und den Descartes scharfsinnig überbrückt hat, durch sein cogito ergo sum, durch sein Ausgehen von dem Geist, der bekannter sei als der Körper, und durch sein Weiterdenken, von der Möglichkeit zur Wirklichkeit der Welt. ${ }^{29}$ ) Zwei andere Gründe führt Cyrano allerdings an, die recht charakteristisch sind. Er sagt: ${ }^{30}$ ) Wir könnten also zu der Überzeugung kommen, unser Leben sei ein beständiger Traum und außerhalb unserer Sinne existiere nichts; aber 1. weil solche Empfindungen in uns unter gewissen Bedingungen immer wieder sich zeigen, und 2. weil andere bezeugen, dieselben Empfindungen zu haben, schließen wir, daß es etwas außerhalb von uns gebe, das Ursache all dieser Erscheinungen sei. " Die Erfahrung also ist es, die Cyrano für fähig hält, uns einen Schluß auf die Existenz der Körper zu erlauben; aber doch nur, fügt er hinzu, wenn die faiblesse de notre raisonnement gestützt werde durch die révélations d'un Dieu, auf den doch alles zurückgehe; - und damit tritt er Descartes wieder näher.

\section{§. Gott.}

In der Tat, auch für ihn war das Dasein Gottes erste und höchste Voraussetzung. Sie ist ihm selbstverständlich, und das Dasein Gottes beweisen zu wollen, macht er nie einen Versuch. Es scheint, als ob man sonst allgemein genejgt ist, Cyrano für einen Atheisten zu erklären; selbst Bouillier ${ }^{31}$ ) ist da über ihn durchaus im unklaren. Ich verweise auf die oben angeführte Stelle Cyr. II, 82; sowie Cyr. II, 368 und Cyr. II, 178: Lettre contre un pédant.

29) Desc. Méd. 6 (I, $322 \mathrm{ff}$.)

30) Cyr. II, 366 .

$\left.{ }^{31}\right)$ Boullier I, 29: La littérature et la poésie du règne de Louis XIII et de la minorité de Louis XIV ont une teinte de licence, d'incrédulité et d'athéisme, qui témoigne tristement de cet état des esprits. Je me borne a citer les noms de Lamothe Levayer, de Naudé, de Gui Patin, de Théophile, de Cyrano de Bergerac, de Saint-Pavin, de Desbarreaux etc. 


\section{Materie und Bewegung ( $\$ \S 8-12)$.}

$\S 8$. Was ist Materie?

Diese Frage zu beantworten, ist nicht so leicht, denn wir werden dabei auf Widersprüche in den Aussagen Cyranos stoßen, die sich kaum beseitigen lassen. Bei der Beantwortung dieser Frage standen sich die Anschauungen Descartes' und Gassendis schroff gegenüber. Ersterer sieht das Wesen der Materie ${ }^{32}$ ) in ihrer Ausdehnung. Letzterer nennt als ihre Haupteigenschaften die Solidität und das Gewicht. ${ }^{33}$ ) Descartes behauptet: die Materie ist divisible à l'infini, es gibt also keine unteilbaren Atome, ${ }^{34}$ ) auf deren Existenz Gassendi das Hauptgewicht legt. ${ }^{35}$ ) Im Fragment de Physique steht Cyrano offenbar auf Descartes' Seite, indem er das Wesen der Materie weder in Härte, noch in Wärme, noch in Farbe, sondern in der Ausdehnung ${ }^{36}$ ) sieht, und indem er als eine der Eigenschaften der Materie ihre Teilbarkeit ins Unendliche nennt. ${ }^{37}$ ) Aber gleichwohl sind die Gegengründe Gassendis nicht ohne Einfluß auf ihn geblieben, denn an einer andren Stelle sagt er ausdrücklich, daß selbst Descartes von dem so erklärten Wesen der Materie keine Rechenschaft ablegen könne. ${ }^{38}$ )

\section{§9. Der leere Raum.}

Bei Descartes ist Ausdehnung und Materie ein und dasselbe, in gewissem Sinne identisch; infolgedessen muß er die Existenz des absolut leeren Raumes, des vide, oder des vacuum leugnen. ${ }^{39}$ ) So tut dies auch Cyrano im Fragment de Physique, ${ }^{40}$ ) und zwar

32) Desc. Princ. Phil. II, 4 (III, 123).

33) Gass. I, 259; 273.

34) Desc. Princ. Phil. II, 20 (III, 137).

35) Gass. I, 257.

36) Cyr. II, 372.

${ }^{37}$ ) Cyr. II, 377.

38) Cyr. I, 407: Ainsi la matière étant divisible à l'infini, il ne faut pas douter que c'est une de ces cboses, qu'il (Descartes) ne peut comprendre, ni imaginer et qu'il est bien au dessus de lui d'en rendre raison.

${ }^{39}$ ) Desc. Princ. Phil. II, 5, 6, 7, 16, 17, 18, 19 (III, 124/5; 133/6). Le Monde ou Traité de la Lumière cap. IV, $231 \mathrm{ff}$.).

40) Cyr. II, 373. 
aus denselben Beweisgründen hervorgehend, die Descartes als maßgebend angibt. An anderer Stelle aber ${ }^{41}$ ) glaubt er einen Widerspruch in Descartes' Leugnen des leeren Raums zu finden, und zwar auf Grund Gassendischer Argumente, die erklären, daß ohne Annahme des leeren Raums keine Bewegung denkbar sei. ${ }^{42}$ ) Diese Schwierigkeit sucht Descartes zu heben ${ }^{43}$ ) dadurch, dass er jede Bewegung als Kreisbewegung (mouvement circulaire) sieht, indem jedes Körperchen, im Kreise sich weiterbewegend, immer an die Stelle eines andren tritt. Indes will Descartes selbst diese Frage nicht endgiltig entscheiden, da die Erfahrungen noch nicht genügendes Beweismaterial gebracht hätten. ${ }^{44}$ ) Auch das wußte (yrano; er sagt: ${ }^{45}$ ) Descartes selbst konnte nicht mehr in Bezug auf diese Frage éclaircir des doutes que la surprise de la mort l'avait contraint de laisser à la terre qu'il venait de quitter. Und auch er läßt hinter der Frage ein non liquet. So ist es zu erklären, daß er an einer andren Stelle ${ }^{46}$ ) offenbar Gassendis Anschauung verteidigt. Wie sehr ihn diese Frage in all ihren Konsequenzen beschäftigte, zeigt dort die lange Auseinandersetzung des Kastilianers, ${ }^{47}$ ) der das Vorhandensein absolut leerer Räume behauptet und ganz in Gassendischen Sinne verteidigt. Brun geht ganz fehl, ${ }^{48}$ ) wenn er hier eine Satire gegen Gassendi zu finden glaubt. Cyranos Ironie kann doch nur Descartes treffen. Brun lässt die oben angeführte Stelle Cyr. I, 406 außer acht; außerdem

41) Cyr. I, 404, 405.

42) Gass. I, 192: Non videtur quippe esse motus, nisi sit Inane.

43) Desc. Princ. Phil. II, 33 (III, 147). Le Monde ou Traité de la lumière cap. IV (IV, 232).

44) Desc. Le Monde ou Traité de la lumière cap. IV (IV, 234): Au reste je ne veux pas assurer pour cela qu'il n'y a point du tout du vide en la nature, ... les expériences ne sont point suffisantes pour le prouver.

${ }^{45}$ ) Cyr. I, 406.

46) Cyr. I, 74: J'ose bien dire, que si il n'y avait point de vide, il n'y aurait point de mouvement, ou il faut admettre la pénétration des corps. Il serait trop ridicule de croire que quand une mouche pousse de l'aile une parcelle d'air, cette parcelle en fait reculer devant elle une autre et qu'ainsi l'agitation du petit orteil d'une puce allât faire une bosse derrière le monde.

47) Cyr. I, $67 \mathrm{ff}$.

48) Brun 306. 
ist ja auch der Rest der Behauptungen des Kastilianers durchaus ernst zu fassen, wie es Brun selbst für den ersten Teil seiner Ausführungen tut.

Das Steigen des Wassers in der Pumpe, das man früher aus dem der Natur eignen horror vacui erklärte, bringt auch Cyrano nicht mehr mit diesem veralteten Begriff zusammen. Seine Erklärung ${ }^{49}$ ) ist durchaus modern und beweist, daß er sich die Resultate der damaligen neuen Entdeckungen auf naturwissenschaftlichem Gebiete zu eigen gemacht hatte; ebenso wie sein Lehrer Gassendi.

\section{$\S 10$. Die Bewegung und die Ruhe.}

Schon in der Definition des Begriffs der Bewegung sieht man, daß hier Cyrano ganz unter Descartes' Einfluß steht. Gassendi nimmt seine Definition von Epikur, seinem anerkannten Meister;

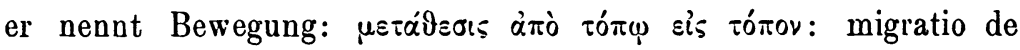
loco in locum. ${ }^{50}$ ) Denn Epikur und mit ihm Gassendi fassen die Materie auf als in dem Rahmen der Ausdehnung oder des Raumes befindlich und sich bewegend. ${ }^{51}$ ) Nach Descartesscher Auffassung dagegen, ${ }^{52}$ ) der, wie wir sehen, auch Cyrano folgt, ist Materie und Ausdehnung dasselbe. ${ }^{53}$ ) Demgemäß mußten sie Gassendis Definition für ungenügend erklären; und nach ihnen besteht die Natur der Bewegung ${ }^{54}$ ) darin, qu'il est le passage d'un corps du voisinage de certains êtres dans le voisinage d'autres êtres.

In derselben Reihenfolge wie bei Descartes folgt jetzt in Cyranos Fragment de Physique die Behandlung der Frage vom Zustand der Ruhe; und wie Descartes kommt er zu dem Resultat, daß man von Ruhe nur in relativem Sinne sprechen könne; denn wenn ein Körper aus der Nachbarschaft des einen in die eines andren sich

49) Cyr. I, 75: étant jointe avec l'air d'une nuance imperceptible, elle (l'eau) s'élève quand on élève en haut l'air, qui la tient embrassée.

50) Gass. I, 338.

51) Gass. I, $229 \mathrm{ff}$.

52) Vergl. § 8.

53) Vergl. § 8 und Cyr. II, 379.

54) Desc. Princ. Phil, II, 24, 25 (III, 139/40). Cyr. II, 378. 
bewegt, so ist der Akt der Trennung beiden gemeinsam. Dieser löst sich sowohl von jenem, wie jener von diesem; der eine bewegt sich also nur mit Rücksicht auf den andren. ${ }^{55}$ ) Ob ich mich um meine Achse drehe, oder ob die Welt sich um mich dreht, in beiden Fällen, sind beide Körper, relativ gefaßt, in Bewegung oder in Ruhe. ${ }^{56}$ ) Diese Ausdrücke gehören also nur dem Sprachgebrauch an, vor dem man sich zu hüten hat. Auch in Bezug auf einen näheren und einen ferneren Gegenstand kann ein Körper gleichzeitig in Ruhe und in Bewegung sein; so ein im $W$ asser schwimmendes Schiff mit Bezug auf das Wasser und mit Bezug auf das Ufer. ${ }^{57}$ ) Es läßt sich höchstens dem Körper bestimmt die Bewegung zuschreiben, indem der Grund der Bewegung liegt.

\section{$\S 11$. Die Gründe der Bewegung.}

Auch bei der Erörterung der Gründe der Bewegung schließt sich Cyrano Descartes an. Neben der ingenerabilitas und incorruptibilitas, Eigenschaften, die Aristoteles schon der Materie beilegte, erkannte Gassendi in den von ihm angenommenen Atomen vor allem Solidität und Widerstandskraft, auch hierin Epikur und Lukretius folgend. Ebenfalls diesen und Demokrits Ansichten gemäß verlegt er schließlich auch das Prinzip der Bewegung in die Materie selbst. Jedes Atom hat von Natur seine Kraft der Bewegung in sich ${ }^{58}$ ) Mit diesem Grundsatz sprach Gassendi einen tief einschneidenden Gegensatz zum Kartesianismus aus. Descartes erkannte der Materie nur die Ausdehnung als Eigenschaft zu; und verlegte den Ursprung jeder Bewegung in Gott. Auch Gassendi stellt sich Gott als den Schöpfer der beweglichen Materie vor, aber nạchdem die Welt einmal in Bewegung gesetzt ist, braucht Gott nicht mehr einzugreifen; während alles was im Kartesianismus

55) Cyr. II, 379.

56) Cyr. II, 380.

${ }^{57)}$ Cyr. II, 381; Desc. Princ. Phil. II, 29.

${ }^{58}$ ) Gass. I, 334: Notum est enim ex antedictis, atomos, quas illi dixerunt esse rerum materiam, habitas iis fuisse non inertes, immobilesque, sed actuosissimas et mobilissimas potius ... 335: Fecisse melius ei videntur, qui agendi principium fecere corporeum ac censuere adeo materiam non inertem, sed actuosam esse. 
den Keim des Okkasionalismus in sich trägt, geradezu dieser Ansicht zuwiderläuft. ${ }^{59}$ ) Der Dualismus Descartes' stellt sich die Materie als kraft- und tatlos dar, ohne den concours ordinaire de Dieu; und gerade letzteren leugnet Gassendi entschieden, und nicht mit Unrecht behauptet er, daß die Ausdehnung allein nicht genüge, um die Natur der Dinge zu erklären. Die moderne Anschauung hat ihm recht gegeben: die Identität von Materie und Ausdehnung ist ein heutzutage nicht mehr maßgebender Grundsatz. ${ }^{60}$ )

In diesem Kampfe steht Cyrano auf Descartes' Seite, wenigstens im Fragment de Physique. Dort spricht er es aus, dass Gott die Welt geschaffen habe, jedem Teilchen der Körper seine Bewegung verleihe, und dass der Bestand aller Dinge in der Welt nur möglich sei durch die fortdauernde Einwirkung Gottes. ${ }^{61}$ ) Wir kommen indes in andrem Zusammenhang auf die Stellung, die Cyrano Gott anweist, zurück. Der Widerspruch, den man an einer andren Stelle ${ }^{62}$ ) gegen das Obengesagte finden könnte, fällt in sich zusammen durch die ironische Auffassung, die man derselben offenbar geben muß.

\section{$\S 12 . \quad$ Es gibt nur eine Materie.}

So lehrte Descartes, ${ }^{63}$ ) und Cyrano folgte ihm auch hierin. Wir sehen wohl Wasser, Feuer und Luft, als verschiedene Dinge, aber in Wirklichkeit sind alle eins und dasselbe; es gibt nur eine Materie, die als ausgezeichnete Schauspielerin in mannigfachem

${ }^{59}$ ) Desc. Princ. Phil. II, 36 (III, 150): Pour ce qui est de la première cause, il me semble qu'il est évident, qu'il n'y en a point d'autre que Dieu, qui par sa toute puissance a créé la matière avec le mouvement et le repos de ses parties, et qui conserve maintenant en l'univers par son concours ordinaire autant de mouvement et de repos qu'il y en a mis en le créant.

$\left.{ }^{60}\right)$ Lotze, Grundzüge der Naturphilosophie, Leipzig 1882. - $\$ 29$.

G. Noodt, Prinzipien der Descartesschen Naturphilosophie, Dissertation. Berlin 1893.

${ }^{61)}$ Cyr. II, 382: S'il ne continuait toujours l'action, par laquelle il nous a tiré du néant, pour nous conserver, nous cesserions d'être tout à coup.

62) Cyr. I, 13.

63) Desc. Princ. Pbil. II, 22 (III, 138): Il n'est pas malaisé d'inférer de tout ceci que la terre et les cieux sont faits d'une même matière. 
Gewand erscheint. ${ }^{64}$ ) Die Erklärungen der verschiedenen Wirkungen der Materie, warum Feuer brennt und Wasser nicht, ${ }^{65}$ ) sind bloßer Ausfluß von Cyranos Phantasie. Aber die Tatsache des tout est en tout steht ihm fest. ${ }^{66}$ ) Allerdings nicht in dem Sinne, wie wir heute es verstehen würden, versucht er nachzuweisen, daß im Wasser Elemente der Erde, in der Erde solche der Luft, im Feuer solche des Wassers u. s. f. sind. Auch an den seltsamen Beispielen, die er anführt, ${ }^{67}$ ) sieht man nicht, welchen Grad von Klarheit seine Vorstellungen in diesem Punkte besaßen. Eins aber zeigen sie sehr deutlich, nämlich, mit welch aufmerksamen Augen Cyrano die Natur betrachtete, mit welchem Staunen er die geschaffenen Dinge und die Wunder der Welt anschaute, und wie sein ganzes Wesen erfüllt war von einer tiefen Liebe zu der Schönheit der Natur. Allen Dingen, leblosen wie lebenden, brachte er ein tiefes Interesse und Verständnis entgegen.

\section{Cyranos Stellung zum Descartesschen Automatismus}

(§§ 13-16).

\section{$\S 13$. Seine Liebe zur Natur.}

Damit kommen wir auf einen Punkt zu sprechen, der der Widerspiegelung Descartesscher Gedanken in Cyranos Kopf eine besondere Färbung gibt. Gassendis Lehren waren doch nicht so ganz spurlos an ihm vorübergegangen, wie es nach dem bis jetzt erwähnten den Anschein haben könnte. Wir sahen, wie er Descartes' Ideen Schritt für Schritt folgt. Aber wo dieselben anfangen, seinem eigenen Wesen zu widersprechen, da machte er einfach Halt und bringt-seine eigenen Gedanken oder jedenfalls solche, die ihm besser passen. So bleibt er doch in etwa seiner Devise treu: Nullius addictus jurare in verba magistri.

Ein Grundzug seines Wesens war nun, wie wir sahen, die Liebe zur Natur. „Hé bien!" ruft er aus, „Vous autres hommes
64) Cyr. I, 68.
65) Cyr. I, 69.
${ }^{66)}$ Cyr. I, 76.
67) Cyr. I, 76-82. 
Vous regardez éternellement les choses, mais Vous ne lès contemplez jamais." Cyrano betrachtete die Natur liebevoll. Er sah überall Leben. Und die von Descartes aus seinem Dualismus gezogene Konsequenz,${ }^{68}$ ) die Körper seien nur Automaten, das Tier sei vernunftlos, mußte ihn in seinem innersten $W$ esen abstoßen.

\section{$\S$ 14. Die Tiere und Pflanzen haben Vernunft.}

Inwiefern sich Cyrano mit dieser Behauptung der Inkonsequenz schuldig machte und in Widerspruch verwickelte mit andern von Descartes übernommenen Ideen, das ist ihm wohl kaum klar geworden. Unbedenklich ging er, seiner Natur folgend, ins feindliche Lager über. Descartes schloß aus der für ihn bewiesenen Tatsache, daß die Tiere nicht sprechen könnten, daß sie keine Vernunft besässen. ${ }^{69}$ ) Dagegen wendet sich Cyrano mit seiner Satire, deren Ernst allerdings meist nicht zu verkennen ist. Und da handelt er als echter und getreuer Schüler Gassendis, der Descartes' Automatismus angriff, ${ }^{70}$ ) und den Mineralien und Pflanzen Sensibilität, den Tieren die Fähigkeit der Sprache zuerkannte. ${ }^{11}$ ) Auf dem Mond und der Sonne lebt alles, die Pflanzen und sogar die Steine.

Schon früher batte die Satire sich oft gegen die gerichtet, die den Tieren die Vernunft absprachen. Montaigne in seiner apologie de Raymond de Sebonde hatte erklärt, daß die Tiere soviel und oft mehr Vernunft zeigen als die Menschen. Hieronymus Rosarius schrieb im 16. Jahrhundert ein Buch, das den Titel führte: Quod animalia bruta saepe ratione utantur melius homine, und das Gabriel Naudaeus 1648 herausgab. ${ }^{72}$ ) Henricus Morus läßt sogar Papageien und Elstern mit Verstand reden. Ähnliches bringt Charron in seinem Buch: De la sagesse I, $8 .{ }^{{ }^{3}}$ ) Dieselbe Bahn wandelt Cyrano. Auf dem Mond wird der Mensch als ein Tier betrachtet, jeder Vernunft bar. Cyrano wird auf dem Mond als Un-

${ }^{68}$ ) Desc. Les passions de l'âme I, 4, 5, 6. L'Homme (IV, 335; 347 ff.).

${ }^{69)}$ Desc. Méth. V (I).

${ }^{70)}$ In der Objection $\mathrm{V}$ gegen Desc. Méditationes (Cousin Ed. II, $108 \mathrm{ff}$.).

71) Gass. II, 328.

72) F. A. Lange, Geschichte des Materialismus. 2 Bde: I, 201.

$\left.{ }^{73}\right)$ Bouillier I, 140 Anm. 
geheuer in einen Käfig gesteckt. ${ }^{74}$ ) Auf der Sonne wird ihm im Reich der Vögel der Prozess gemacht, ${ }^{75}$ ) weil er zu der Kategorie von Wesen gehöre, die sich Menschen nenne, die sich einbilde, die Welt sei für sie da, und sie besäßen Vernunft. Hé quoi, murmeln die Vögel hinter seinem Rücken, il n'a ni bec, ni plumes, ni griffes, et son âme serait spirituelle! oh, Dieux, quelle impertinence! ${ }^{76}$ )

Ein ganzer Abschnitt des „royaume des oiseaux" enthält nur „Plaidoyé au parlement des oiseaux, les chambres assemblées, contre un animal, accusé d'être homme. ${ }^{77}$ ) Und vor diesen Vogelrichtern weiß sich Cyrano nur dadurch zu helfen, daß er sich für einen Affen ausgibt. ${ }^{78}$ ) Aber selbst damit überlistet er die Vögel nicht, denn die sind viel zu klug. Sie halten einen Menschen für quelque chose de si abominable, qu'il était utile qu'on crût que ce n'était qu'un être imaginaire. ${ }^{79}$ ) Ja, der Staatsanwalt im Reich der Vögel erklärt être né homme sei gleichbedeutend mit dégradé de la raison et de l'immortalité. ${ }^{80}$ )

Die Bäume haben dieselben geistigen Fähigkeiten wie die Menschen, sie lieben und hassen ebenso wie diese. ${ }^{81}$ ) Ja, der Kohlkopf ist dem Menschen bedeutend überlegen. In einer langen Auseinandersetzung, ${ }^{82}$ ) die an eine ganz ähnlicheStelle bei Gassendi ${ }^{83}$ ) erinnert, vergleicht Cyrano Mensch und Kohlkopf. Das Resultat ist: le chou nous est superieur par un intellect universel, une connaissance parfaite de toutes les choses dans leurs causes. ${ }^{84}$ ) Wenn. Moses vom Baume der Erkenntnis spricht, so will er damit nur sagen, dass die Pflanzen die vollkommene Philosophie besitzen. ${ }^{85}$ )
74) Cyr. I, 40.
${ }^{75)}$ Cyr. I, $316 \mathrm{ff}$.
${ }^{76}$ ) Cyr. I, 319, 320.
$\left.{ }^{77}\right)$ Cyr. I, 334.
78) Cyr. I. 321.
$\left.{ }^{79}\right)$ Cyr. I, 322.
${ }^{80}$ ) Cyr. I, 334.
${ }^{81)}$ Cyr. I, $361 \mathrm{ff}$.
${ }^{82)}$ Cyr. I, 113-119.
$\left.{ }^{83}\right)$ Gass. II, 556.
84) Cyr. I, 117.
85) Cyr. I, 118. 
Es ist nicht schwer, aus all diesem die Satire herauszulesen, deren Hauptangriffspunkt ist: die Tiere seien vernunftlos. Wir deuteten schon an, daß hier Cyrano auf Gassendis Seite steht, der allen Dingen auf der Erde eine gewisse intellektuelle Fähigkeit zusprach. ${ }^{86}$ )

\section{$\S 15$. Die Tiere und Pflanzen sprechen.}

Descartes hatte den Tieren die Fähigkeit der Sprache abgestritten ${ }^{87}$ ) Gassendi dagegen erklärt, ${ }^{88}$ ) unsere Sprache hätten die Tiere allerdings nicht, aber eine Art und Weise des Ausdrucks, mit der sie sich unter sich selbst verständigen, und der, falls wir die nötigen Organe hätten, auch wir zu folgen imstande wären, ließe sich ihnen jedenfalls nicht abstreiten. Demgemäß stattet auch Cyrano all seine Tiergestalten mit Sprache aus. Die Vögel sprechen, flötend und singend. ${ }^{89}$ ) Daß die Bäume auf der Sonne griechisch sprechen ${ }^{90}$ ) hat nichts Wunderbares, stammen sie doch von den Eichen des Heines von Dodona in Epirus, die schon den Griechen ein heiliges Orakel waren. Die Sprache der anderen Bäume ist der leise Hauch und das Rauschen, das durch ihre Blätter geht. ${ }^{91}$ )

\section{$\S 16$. Die Allbeseeltheit der Welt.}

Gassendi sprach den Dingen nicht nur eine Art anima zu; er erkannte auch eine geistige Verbindung zwischen allen Erscheinungen der Welt an.

In Übereinstimmung mit der vorsokratischen Philosophie, und wohl in engerer Anlehnung an naturphilosophische Ideen der Renaissance hielt er die Welt für Ě $\mu \Psi u \times o \varsigma$ xai vospós.

$\left.{ }^{86}\right)$ Gass. II, 3: Ceterum autem, si cum anima tribuatur vulgo solum plantis et brutis ac hominibus, id non obstat quominus videatur plerisque attribuenda esse metallis et lapidibus.

${ }^{87}$ ) Desc. Méth. V.

88) Gass. II, 522, 523.

${ }^{89}$ ) Cyr. I, 312, 289-90.

${ }^{90)}$ Cyr. I, 352-55.

91) Cyr. I, 357. 
In dem Widerstand der schweren Körper, sich von der Erde zu trennen, in der Anziehungskraft, war er geneigt, eine Art von Gefühl, eine bewußte Kraft anzunehmen. ${ }^{92}$ ) In den Atomen muß ein gewisser Geist stecken, der sie bewusst regiert, den wir zu erkennen aber nicht imstande sind. Die ganze Welt ist beseelt..$^{93}$ ) Daran dachte offenbar Cyrano, wenn er verschiedentlich von Dingen redet, die wir in unserer Zeit mit dem Namen Telepathie bezeichnen würden. Und es läßt sich nicht leugnen, daß trotz ihres phantastischen Anstrichs diese Allbeseeltheit der Welt, wie sie Cyrano schildert, soviel natürlicher und sinnreicher ist als etwa die Märchengeschichten von den Saganen desBombastus Theophrastus Paracelsus. ${ }^{94}$ ) Campanella, der nach seinem Tode auf der Sonne im Lande der Philosophen lebt, weiß von der Ankunft Descartes' schon, obgleich dieser noch drei Meilen von ihm entfernt ist. Es nimmt sich dann im folgenden äußerst seltsam aus, wie Descartes, der wirklich bald darauf erscheint, dem erstaunten Cyrano die Erklärung dieser Tatsache ganz in Gassendischem Sinne gibt: Die Körper atmen gewisse Atombilder aus, die Form und Proportion des Gegenstandes trotz ihrer Bewegung behalten. Auf der Erde, wo die Seele in der groben Materie des menschlichen Leibes eingeschlossen ist, kommt die Wirksamkeit dieser Atome nur selten zu tage; aber hier auf der Sonne empfinden wir es, wenn sie sich in unsere Seele einprägen und uns ferne Dinge mitteilen, die die fünf Sinne nicht erfassen können. ${ }^{95}$ ) Ähnlich ist die Erklärung für die Erscheinung, daß man durch bloßes Anpassen der Bewegung und Lage der Glieder, sowie durch blosses Nachahmen der Gesten und Mienen eines anderen Wesens dieselben Gefühle und Ideen nachzuempfinden und nachzubilden im stande sei, die dies andere Wesen in dem betreffenden Augenblick habe; denn dieselbe

92) P. F. Thomas: La philosophie de Gassendi, p. 111.

93) Gass. II, 3: cur non possit quoque attribuendo [anima] videri terrae? Equid repugnat esse in terra genus vitae et cognitionis, quod nobis Homuncionibus et Terrigenis, seu pusillimis particulis terrae assequi non liceat.

94) Rixner und Siber, Leben und Lehrmeinungen berühmter Physiker am Ende des 16. und Anfang des 17. Jahrhunderts. Sulzbach 1819-26. Heft I, 71-73.

95) Cyr. I, 442-444. 
Disposition der Materie müsse natürlicherweise dieselben Gedanken produzieren..$^{96}$ )

In der Tat, Cyrano ist hier weit von dem Dualismus Descartes' entfernt. Es wird kaum möglich sein, diese Widersprüche völlig miteinander auszusöhnen. Wo sah er selbst die Wahrheit, wenn er an der einen Stelle sich so, an der anderen entgegengesetzt erklärt. Begnügen wir uns vorläufig mit der Tatsache, daß Cyrano trotz seiner Verfechtung Descartesscher Theorien auch mit Gassendis Atomenlehre hinreichend vertraut war, um dies an manchen Punkten zu zeigen. ${ }^{97}$ )

\section{Erklärung der Arbeit der Sinnesorgane (\$§ 17-21).}

\section{$\$ 17$. Das Sehen.}

So erklärt er auch die Arbeit der Sinnesorgane durch die Bewegung unteilbarer Atome ${ }^{98}$ ).

Descartes erklärt das Sehen durch Lichtteilchen, die von dem hellen Körper fortgeschleudert werden ${ }^{99}$ ). Ganz anders Cyrano. Er sagt ${ }^{100}$ ), die Häutchen der Augen lassen einen Feuerstaub durch, der aus den Augen kommt, und den man Gesichtsstrahlen nenut. Werden diese Strahlen durch einen undurchsichtigen Gegenstand aufgehalten, so kehren sie zum Auge zurück, nicht ohne die unzählige Menge kleiner Atome, die das Bild des betreffenden Körpers bilden, mit zum Auge zurückzureißen. Woher hat Cyrano diese seltsame Theorie? Von Descartes nicht, wie wir sahen; aber auch nicht von Gassendi. Dieser entschied sich in dem Streit: an visio fiat per emissionem cuiuspiam rei ex oculis,

96) Cyr. I, 388, 389 .

${ }^{97)}$ Cyr. I, $131 \mathrm{ff} ., 159,160$.

$\left.{ }^{98}\right)$ Cyr. I, 163.

${ }^{99}$ ) Desc. Dioptr. (III, 3). Je désire que Vous pensiez que la lumière n'est autre chose dans les corps, qu'on nomme lumineux, qu'un certain mouvement ou une action fort prompte et fort vive, qui passe vers nos yeux par l'entremise de l'air et des autres corps transparents. Desc. Dioptr. (III, 9) Pour l'ordinaire des hommes, ils ne voient que par l'action, qui vient des objets; car l'experience nous montre que ces objets doivent être illuminés, pour être vus, et non point nos yeux pour les voir.

100) Cyr. I, 136. 
an vero potius per receptionem cuiuspiam rei ex ipsis rebus? für die letztere Alternative, indem er der Bilderhypothese Epikurs, Demokrits und der jüngeren Peripatetiker folgte. Diese behaupteten, aus den Körpern strömten quaedam simulacra derselben aus, allerdings nicht, wie Aristoteles erklärte: formae sine materia, sondern aus Atomen bestehend; diese treffen das Auge und erregen den Sehvorgang ${ }^{101}$ ). Diese simulacra corporum sind offenbar das, was Cyrano l'image de l'objet nennt ${ }^{102}$ ). Und wenn er dies erklärt: cette image n'étant qu'un nombre infini de petits corps qui s'exhalent continuellement en égale superficie de l'objet regardé $\left.{ }^{103}\right)$, so ist das nichts andres als die Erläuterung Epikurs: $a b$ omnibus corporibus ingi fluore quaedam simulacra manare ${ }^{104}$ ).

Aber während Gassendi ausdrücklich sagt ${ }^{105}$ ): videtur imprimis nihil esse necesse, ut quidpiam ex oculis prodeat quod in res visas emittatur, verbindet die Theorie Cyranos beide oben angeführten Alternativen: Vom Auge gehen Strahlen aus, diese werden vom Körper zurückgeworfen, und reißen die simulacra mit. Eine Verbindung beider Anschauungen findet sich schon bei Galenus, bei den Stoikern Chrysippus und Apollodorus und bei Hipparch ${ }^{106}$ ); aber nirgend so, wie sie sich Cyrano zurechtgelegt hat.

\section{§ 18. Das Hören.}

Wie kommt es, daß wir einen Ton hören? Kommt aus den Ohren ein Schwamm, der die Musik trinkt ${ }^{107}$ ), um sie zurückzubringen? Der Harfenspieler, der die Seiten seines Instrumentes schlägt, setzt dadurch die kleineren Luftteilchen, ces petits riens

101) Gass. II, 374 [Plutarch: simulacrorum insinuatio; Cicero: idolorum incursio].

102) Cyr. I, 137.

103) Ebenda.

104) Gass. II, 374.

105) Gass. II, 375.

106) Gass. II, 372, 373: arbitrati sunt prodire ex oculis radios aërem usque ad rem visam sic procudentes intendentesque, ut inde fiat veluti conus, cuius cuspis sit in superficie oculi et in ipsa re visa; ac veluti manus baculo admoto sentit quodam, ... sic visum ... sentire putant.

${ }^{107}$ ) Cyr. I, 139. 
corporels, in Bewegung und jagt sie durch das Ohr in das Gehirn ${ }^{108}$ ). Ist der Ton hoch, so waren die Luftteilchen heftig erschüttert, und umgekehrt.

\section{$\S 19$. Das Tasten.}

Nimmt man an, que de toute matière palpable, il se fait une emission perpetuelle de petits corps, so ist die Erklärung des Tastgefühls einfach ${ }^{109}$ ). Das Organ des Tastens ist auf der ganzen Oberfläche des Körpers verbreitet ${ }^{110}$ ). Dem fügt Cyrano zu: je näher das Glied, mit dem wir tasten, dem Kopfe ist, desto eher unterscheiden wir, was der berührte Gegenstand ist. Die Erklärung, die er hiefür gibt, ist recht phantasiereich: Unsere Haut hat überall kleine Poren und Löcher; und durch diese verlieren die Nerven, die das Tastgefühl zum Gehirn transportieren, eine um so größere Zahl der empfangenen Atome, je ferner der Endpunkt der Reise ist $\left.^{111}\right)$.

\section{$\S 20$. Das Schmecken.}

Wir schmecken eine Frucht, weil dieselbe im Mund eine, je nach der Frucht verschiedene, Art von Salz auflöst und in die Nerven von Gaumen und Zunge dringen läßt ${ }^{112}$ ).

\section{$\S 21$. Das Riechen.}

Der Geruch entsteht durch beständige Emission kleiner Körper von seiten des betreffenden Gegenstands ${ }^{113}$ ).

\section{Schöpfung und Entwicklung der Materie ( $\$ 22$ und 23).}

\section{$\S 22$. Die Ewigkeit der Welt.}

Für die Philosophie des 17. Jahrhunderts gab es eine Autorität, mit der sie rechnen muste, und vor deren Tyrannei sie sich

108) Ebenda; vergleiche dazu: Gass. II, 363: aer a sonante corpore compulsus suoque modo figuratus, ubi est ingressus in aurem, apellit primum ad tympanum etc.

109) Cyr. I, 141.

110) Ebenda, vergleiche dazu: Gass. II, 353: esse ipsi tactui sensorium toto diffusum corpore.

111) Cyr. I, 142.

112) Cyr. I, 142; vergleiche dazu: Gass. II, 357.

113) Cyr. I, 143; vergleiche dazu: Gass. II, 260. 
mehr als einmal beugte. Das war die Kirche. Man suchte möglichst jeden Konflikt mit ihr zu vermeiden, denn sie hatte die Macht. Die Widersprüche, die sich zwischen kirchlichen Dogmen und philosophischen Grundsätzen und Erkenntnissen herausstellten, versuchte man mit krampfhaftem Bemühen aufzuheben. Die Behauptung von der Ewigkeit der Welt widersprach aber der christlichen Schöpfungsgeschichte.

Xenophanes, Parmenides, Melissus der Chaldäer(?), Pythagoras, Plato und Aristoteles lehren, so sagt Gassendi ${ }^{114}$ ), die Welt habe keinen Anfang gehabt und sei ewig. Empedokles dagegen, Heraklit, Anaximander, Anaximenes, Anaxagoras, Archelaus, Diogenes von Apollonien, Leukippus, Demokritus, Epikur, Zeno und die Stoiker erkennen der Welt einen Anfang zu. Gassendi selbst, getreu Epikur folgend, schließt sich letzterer Ansicht an. Er bleibt so also auch in Übereinstimmung mit Dogma und Kirche, zumal er ausdrücklich Gott als den Schöpfer hinstellt ${ }^{115}$ ).

Cyrano folgt auch in diesem Punkte Gassendi nicht. Obwohl er an einer Stelle sagt, daß in solchen Dingen die Vernunft vor dem Glauben zurückstehen müsse ${ }^{116}$ ), und an einer anderen die Ewigkeit der Welt für "une rêverie contraire à ce que la foi nous apprend erklärt ${ }^{117}$ ), gibt er doch gleichzeitig zu, daß seine lebhafte Einbildungskraft sich bei dieser Entscheidung nicht beruhigt hat $\left.{ }^{118}\right)$. Bei dem kirchlichen Dogma bleibt ihm der Ewigkeitsgedanke le premier obstacle, qui nous arrête ${ }^{119}$ ). Der menschliche Verstand sei allerdings nicht stark genug, um diese gewaltige Idee in sich aufzufassen und zu begreifen, wie dies große, schöne und so wohl geordnete Universum ohne Schöpfung habe entstehen können. Wir verstehen die Ewigkeit der Welt nicht; und deshalb schreiben wir die Schöpfung einem Gott zu; aber sind wir so nicht „semblable à celui, qui s'enfoncerait dans la rivière de peur d'être

\footnotetext{
114) Gass. I, 162.

${ }_{115)}$ Gass. I, $163 \mathrm{ff}$.

${ }^{116)}$ Cyr. I, 18.

117) Cyr. I, 96.

118 ) Cyr. I, 18.

119) Cyr. I, 130.
} 
mouillé de la pluie $\left.{ }^{(120}\right)$. Wie will man, fragt er sich verzweifelt, den Schritt vom Nichts zum Atom erklären, der so gewaltig ist, dass „la cervelle la plus aigue n'y saurait pénétrer ${ }^{121}$ ). Um sich aus diesem verwirrenden Labyrinth zu retten, gibt es nur einen Ausweg: zugleich mit Gott eine ewige Materie anzunehmen.

\section{§23. Evolutionsgedanken.}

Wenn schon im Vorigen Cyrano seinen eigenen Weg ging, so noch mehr in einem anderen Punkte, nämlich in der Frage: Wie hat man sich dann weiter die Fortentwickelung der Materie bis zu den so mannigfaltig variierten Phänomenen der Natur zu erklären?

Die kirchliche Schöpfungsgeschichte lehrt, dass Gott die Welt von Anfang an in ihrer endgültigen Form schuf. In dem Sinne spricht sich auch Descartes ${ }^{122}$ ) aus (Méth. V), obgleich er selbst viel mehr zu Entwicklungsgedanken hinneigte, als dort zu ersehen ist. Nur in einem Satz drückt er sich vorsichtig so aus, dass man das Wesen der Welt wohl leichter verstehen könne, wenn man sie in ihrer allmählichen Entwicklung betrachte, als wenn man sie als schlechthin gegeben und fertig ansehe. Seine Zurückhaltung ist wohl ausschliesslich auf die abschreckende Wirkung von Galilei's Verurteilung zurückzuführen, und auf das Bestreben Descartes', jede Kollision mit der geoffenbarten Religion zu vermeiden. ${ }^{123}$ )

Aber Cyrano beruhigte sich bei der einmaligen, endgültigen Schöpfung aller Dinge durch Gott nicht. Die Art und Weise allerdings, wie er sich das gewaltige Rätsel der Entwicklung vom Atom bis zur Mannigfaltigkeit der Dinge zu erklären sucht, war gewiss auch für ihn selbst nur ein Produkt seiner Phantasie. Aber der Gedanke beherrscht ihn; und unbewusst nähert er sich einer uns heute ganz vertrauten Theorie, nämlich der Evolutions- oder Transformationstheorie.

120) Ebenda.

121) Cyr. I, 131.

$\left.{ }^{122}\right)$ Desc. Méth. V (I).

${ }^{123}$ ) Cf. Übersetzung des Disc. d. l. méth. von Dr. L. Fischer. S. 63. Anmerkung. 
P. A. Brun hat zuerst darauf hingewiesen ${ }^{124}$ ), und offenbar mit Recht, wenn auch sein Versuch, Cyrano in eine Reihe mit Darwin, Haeckel, und Spencer zu stellen, übertrieben ist. Die creatio spontanea war ein damals viel diskutiertes Thema. ${ }^{125}$ ) Sie mag auch bei Cyrano zur Bildung manchen Gedankens massgebend gewesen sein. Nichtsdestoweniger bleibt seine Idee eine beachtenswerte. Er nimmt eine stufenweise Entwicklung der Materie an, vom einfacheren zum komplizierteren Geschöpf, mit dem Prinzip der Vervollkommnung. ${ }^{126}$ )

Von der Gassendi'schen Lehre ausgehend, dass die verschiedene Zahl von verschieden geformten Atomen der Grund der Verschiedenheit der Erscheinungen der Materie sei legt Cyrano der Materie von Anfang an die Absicht bei, den Menschen zu formen; und es ist ihm sehr klar, dass der Weg zu diesem Ziel nur durch unendlich viele und mannigfache Formen führt. ${ }^{127}$ ) Ein gewisser Ernst lässt sich seinen Ausführungen nicht abstreiten, wenn auch gelegentlich bei Anwendungen der Theorie, wieder die Phantasie die Oberhand gewinnt. 'So schildert er, wie auf der Sonne vor seinen Augen ein wunderschöner Granatapfel sich in ein Lebewesen verwandelt ${ }^{128}$ ); oder, infolge der zuerst langsamen, dann rasend schnellen tanzenden Bewegung von Tausenden kleiner lebendiger Wesen entsteht allmählich ein grosser Mensch, bei dem man natürlich die vorher deutlich zu erkennende Bewegung der kleinen Wesen nicht mehr unterscheiden kann. ${ }^{129}$ )

124) Brun, $306 \mathrm{ff}$.

${ }^{125}$ ) Cf. Gass. II, 260: De animalibus sponte nascentis. Desgl. M. de Lanessan, le transformisme, l'évolution de la matière et des Etres vivants.

${ }^{126}$ ) Cyr. I, 156: Tous les êtres dans la nature tendent au plus parfait; et aspirent à devenir hommes.

${ }^{127}$ ) Cyr. I, 133: Un peu moins de certaines figures, c'eût été un orme, un peuplier, un saule; un peu moins de certaines figures c'en̂t été la plante sensitive, une huître à l'écaille, un ver, une mouche, une grenouille, un moineau, un singe, un homme. I, 134: Un million de fois cette matière s'acheminant au dessein d'un homme, s'est arrêté à former tantôt une pierre, tantôt du plomb, tantôt du corail, tantôt une fleur, tantôt une comète; et tout cela à cause du plus ou du moins de certaines figures, qu'il fallait, ou qu'il ne fallait pas, à designer un homme.

${ }^{128}$ ) Cyr. I, 285.

129) Cyr. I, 291; vergl. auch Cyr. I, 156. 


\section{Kosmologische Gedanken ( $\$ \$ 24-27$ ).}

$\S 24$. Die Sonnenflecken.

Einen großen Platz in den phantasiereichen Ausführungen Cyranos nehmen kosmologische Betrachtungen ein, wie dies ja bei Reisen nach Sonne und Mond nicht wundernehmen kann.

Und gerade diese sind wohl am meisten rein gehalten von allzu seltsamen Ausschmückungen; besonders in bezug auf die Sonnenflecken und die Bewegung der Himmelskörper sind seine Ansichten im großen und ganzen richtig.

Die Kraft, die die Entwicklung des Weltalls bewirkt, ist das Feuer $\left.{ }^{130}\right)$; ein Gedanke, der schon von Gassendi wieder aufgenommen worden war. Für die Art der Entstehung der Welt sind die Sonnenflecken die wichtigsten Momente. Das, was wir mit bloßem Auge nur als gewisse dunkle Flecke sehen, sind in Wirklichkeit, wie scharfe Fernrohre beweisen, des mondes, qui se construisent. ${ }^{131}$ ) Ebenso, wie das Feuer die Asche, die es zu ersticken droht, wegschleudert, und wie der Körper durch Erbrechen Verdauungsstörungen beseitigt, so entledigen sich die Sonnen jeden Tag von den Resten der Materie, die ihr Feuer nährt. ${ }^{132}$ ). Dieser Schaum der sich reinigenden Sonne ist der erste Ursprung der die Sonne umgebenden Himmelskörper. ${ }^{133}$ ) . Vielleicht zehrt sich so die Sonne allmählich auf, und wird in unabsehbarer Zeit vielleicht un globe opaque comme la terre. $\left.{ }^{134}\right)$ All diese Fragen waren damals zeitgemäß. Im Hotel de Rambouillet bildeten sie einen

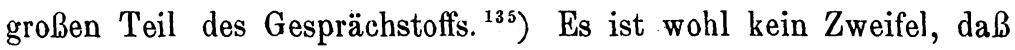
sich Cyrano in bezug auf die Lehre von den Sonnenflecken an Descartes ${ }^{136}$ ) anschließt.

130) Cyr. I, 133: le feu est le constructeur des parties et du tout de l'univers.

131) Cyr. I, 21.

132) Cyr. I, 19.

133) Cyr. I, 18.

${ }^{134}$ ) Cyr. I, 257.

135) Brun, 302.

${ }^{136}$ ) Desc. Le Monde. 
$\S 25$. Die Bewegung der Himmelskörper.

Aber nicht so in bezug auf die Theorie der Bewegung der Himmelskörper, wo Descartes eine Konzession an die kirchliche Lehre machte, Cyrano dagegen frei der Ansicht das Wort redete, die sicherlich auch Descartes in seinem Innern als die richtige anerkannte. Das Bild, das sich die älteste Philosophie vom Weltall machte - die Erde unbeweglich inmitten der sie umgebenden Welt, in der Form einer Scheibe, von dem großen Himmelsgewölbe überdeckt - hatte noch im griechischen Altertum anderen, aufgeklärteren Anschauungen weichen müssen. Das philolaische System der Pythagoreer setzte das reinste und geistigste Element, das heilige Feuer, die Hestia, in die Mitte der Welt; und um dies bewegten sich nach ihrer Anschauung Erde und Gegenerde. Aristarch von Samos führte diese kühne Idee weiter aus ${ }^{137}$ ), widerlegte dagegen erhobene Einwürfe und stellte ein heliozentrisches System auf. ${ }^{138}$ ) Aber dauernde Anerkennung gewannen diese kühnen Spekulationen nicht. Die alexandrinischen Gelehrten, vor allem Ptolemäus, und mit ihnen das gesamte Mittelalter hielten an dem alten aristotelischen, geozentrischen Sphärensystem fest. Die allmählich sich ergebenden Unzulänglichkeiten und die Ungleichheit der Planetenbewegung suchte man vergeblich durch Annahme neuer Sphären und exzentrischer Kreise, und durch die Theorie der Epizyklen zu beseitigen. Der heilige Augustinus sogar, von dem Cyrano bewundernd sagt: ce grand personnage dont le génie était fort éclairé, erklärt: Die Erde sei platt wie ein Ofen und schwimme auf dem Wasser, wie die Hälfte einer durchgeschnittenen Apfelsine. Und noch Melanchthon hielt die Neuerung des Kopernikus für eine „neue so böse und gottlose Meinung“, daß er die Obrigkeit zur Unterdrückung derselben anrief.

137) Vergl. Genaueres: W. Förster, Sammlung wissenschaftlicher Vorträge. Berlin 1876. I, S. 15 ff.: Die Astronomie des Altertums und des Mittelalters im Verbältnis zur neueren Entwicklung (Febr. 1860). Desgl. Lange (ed. 1866) S. 63. Desgl. William Whewell: History of the inductive sciences, London 1847. 3 vols ; cf. I, $159 \mathrm{ff}$., 264, $388 \mathrm{ff}$.

138) Kuhlenbeck, Giordano Brunos Dialoge (de l'infinito, universo e mondi) übersetzt und mit Anmerkungen versehen von Dr. Leo K. Leipzig. IV. 
Das Verdienst, den Kampf gegen das alte komplizierte und unnatürliche System nach Aristarch wieder aufgenommen zu haben, knüpft sich an die Namen: Cusa, Kopernikus, Bruno und Galileï. Kopernikus setzte die Sonne, die Weltleuchte, die die ganze Familie kreisender Gestirne lenkt, in die Mitte des schönen Naturtempels, wie auf einen königlichen Thron. ${ }^{139}$ ) Aber jenseits der Sonne und der Planeten ließ er, und noch Kepler, den unbeweglichen Fixsternhimmel stehen, als Grenze der Welt. Giordano Brunos Feuergeist riß auch diese letzte Schranke nieder und erkannte die Sonnennatur der Fixsterne, die den Blick in die Unendlichkeit des Weltalls freigab.

Descartes fand einen Ausweg, um in dieser Frage jeden Streit mit den Theologen zu vermeiden. Seine Übereinstimmung mit der Kirchenlehre war allerdings nur dem Wortlaut nach vorhanden. Er behauptet: die Erde ruhe, nämlich im Hinblick auf ihre nächste Umgebung, da sie in der den Himmelsraum erfüllenden flüssigen Materie schwimme. ${ }^{140}$ ) So bewegt er die Erde, ohne ihr selbst Bewegung zu verleihen.

Gassendi ${ }^{141}$ ) und mit ihm Cyrano folgen ganz den neuen Ideen. Die Sonnen - unsere Sonne und die anderen Fixsterne sind die Zentren von Planetensystemen. ${ }^{142}$ ) Die Planeten bewegen sich um die Sonne - so unsere Erde um unsere Sonne -, und um sich selbst. Unsere Erde erscheint also dem durch die Luft zur Sonne sausenden Cyrano nur als ein Stern, wie jeder andere, zuletzt nur noch als kleiner leuchtender Punkt, bis auch dieser schließlich ganz aus seinen Augen verschwindet. ${ }^{143}$ ) Die Erde dreht sich um die Sonne und gleichzeitig um sich selbst; und zwar von Osten nach Westen, so sagt Cyrano allerdings irrtümlicherweise an einer Stelle. ${ }^{144}$ )

139) Giordano Brunos Reformation des Hinmels (Spaccio della bestia trionfante) übers. von Dr. L. Kuhlenbeck 1889. IV.

${ }^{140}$ ) Desc. Princ. Phil. III, $140 \mathrm{ff}$. (III, $\left.517 \mathrm{ff}.\right)$.

141) Gass. I, 618.

142) Cyr. I, 15.

143) Cyr. I, 271.

144) Cyr. I, 253-4. 
An einer anderen jedoch legt er die gegenteilige Behauptung der Erzählung zu Grunde, daß er mit seiner Flugmaschine zuerst in Kanada niedergefallen sei. ${ }^{145}$ ) Dort hat er, dies sei kurz erwähnt, mit einem Jesuitenfreund eine lange Auseinandersetzung über die Vernünftigkeit des kopernikanischen Weltsystems. Die Sonne, ohne deren Licht und Wärme kein Wesen bestehen könne, lasse sich schlechterdings nur im Zentrum der um sie sich drehenden Welt denken. ${ }^{146}$ )

Die Planeten haben nur reflektiertes, kein eigenes Licht. ${ }^{147}$ ) Um die Planeten bewegen sich wiederum Himmelskörper, die Trabanten, so um die Erde der Mond. ${ }^{148}$ ) Der Jupiter hat ihrer sogar vier. ${ }^{149}$ )

\section{$\S 26$. Deren Ursache.}

Die Ursache all dieser Bewegungen hatte Descartes durch seine Theorie der Tourbillonen erklärt.

Gassendi, der ja auch seinen Atomen eigene Bewegungsfähigkeit zuschreibt, legt auch den Himmelshörpern selbst die Kraft der Bewegung bei ${ }^{150}$ ). Cyranos Erklärung, obwohl phantastisch, ist insofern beachtenswert, als sie erkennen läßt, daß er in den Bewegungen der Planeten doch eine Abhängigkeit von der Sonne und besonders ihrer Wärme feststellen wollte: die Sonnenstrablen treffen mit all ihren wirkenden Kräften auf die Erde, und bringen sie zur Drehung, wie wir durch einen Schlag der Hand den Globus ${ }^{151}$ ). Bei der Gelegenheit wird erzählt, wie ein Jesuit die Erdbewegung erklärt: das Höllenfeuer ist im Inneren der Erde; die Verdammten, die ihm enfliehen wollen, klettern an der inneren Kugelwölbung in die Höhe, und drehen so die Erde, wie ein Hund ein Rad, in dessen Inneren er läuft ${ }^{152}$ ).

145) Cyr. I, 9.

146) Cyr. I, 11, 12.

147) Cyr. I, 255.

${ }^{148)}$ Desc. Le Monde X (IV, 288).

${ }^{149)}$ Cyr. I, 256.

${ }^{150}$ ) Gass. I, 638: Itaque planius longe videtur asserere caelos siderumve globos moveri ab interno principio, a propriis formis, a se ipsis.

151) Cyr. I, 13.

152) Cyr. I, 14. 


\section{$\S 27$. Der Unendlichkeitsgedanke.}

Mit der Erklärung, die Fixsterne sind Sonnen, gewann die Idee der Unendlichkeit, die schon die alten Griechen beschäftigt hatte, neue

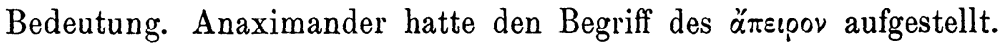
Anaxagoras und die Eleaten versuchten trotz der darin liegenden Widersprüche eine positiv gedachte Unendlichkeit festzuhalten. Gegen sie hatte sich Aristoteles gewandt, mit seiner Kritik dieses Begriffs ${ }^{153}$ ); „von dem Unendlichen, sagt er, ist es ebenso schwer zu behaupten, da $\beta$ es sei, als daß es nicht sei." Er bestreitet die Wirklichkeit eines räumlich unendlichen Seins. Die nacharistotelische Philosophie, insbesondere die Neuplatoniker beschäftigten sich mit Vorliebe mit der Mystik des Seienden Unendlichen. Nikolaus von Cusa spricht dem Weltall unbegrenzte Ausdehnung in Raum und unendliche Dauer in Zeit zu. Insbesondere bei Giordano Bruno bekam der Begriff, wie wir sahen, neues Leben. Descartes, dem die Negativität des Unendlichen ein unklarer Begriff schien, versuchte sich dadurch zu belfen, daß er das All. zwar nicht für unendlich (infinitum), aber für endlos (indefinitum) erklärte ${ }^{154}$ ). Diesen Ausweg macht ihm Cyrano im Fragment de Physique nach ${ }^{155}$ ). Dagegen an andrer Stelle erklärt er dem Vizekönig von Kanada schlechtweg, das Weltall sei unendlich; denn die Bewohner der Fixsternplaneten erkennten sicherlich von ihrer Welt aus wiederum andere neue Sonnensysteme, und so weiter ohne Ende. Auch die weitere Argumentierung ${ }^{156}$ ) entspricht G. Brunos Denkweise, die sich Gott als die dem Universum immanente erste Ursache vorstellt. Denn, sagt Cyrano, vorausgesetzt, die Welt ist nicht unendlich, so wäre ja auch Gott als endlich zu denken, da er ja nicht da sein könne, wo nichts sei. Wie unverständlich auch diese Unendlichkeitsidee sei, man müsse doch an ihr festhalten, erklärt er schließlich; sowie auch Cusa die unendliche Einheit nur durch die visio sine comprehensione erkennt.

\footnotetext{
153) Kublenbeck: G. Brunos Dialoge XXI Einleitung.

${ }^{154)}$ Desc. Princ. Phil. II, 21 (III, 138).

155) Cyr. II, 375.

${ }^{156)}$ Cyr. I, 17.
} 
Also auch hier folgt Cyrano Gassendi nicht, denn ein Teil der Objectiones des Gassendi gegen die Descartesschen Meditationes bestand in dem Angriff gegen die Unendlichkeitslehre, die Descartes vor allem als Gottesbeweis aufgenommen batte ${ }^{157}$ ). Gassendi sagt dort: Celui, qui dit une chose infinie, attribue à une chose qu'il ne comprend pas, un nom qu'il n'entend pas non plus ${ }^{158}$ ).

\section{Schlufsbemerkungen ( $\$ \S 28-30$ ).}

\section{$\S 28$. Die Bewohnbarkeit der Himmelskörper.}

$\mathrm{Ob}$ der Mond und die Planeten Erden wie die unsere und bewohnbar seien, war eine Frage, die schon oft die Aufmerksamkeit der Gelehrten auf sich gezogen hatte. Die Peripatiker lehrten, der Mond sei keine Erde, wie die unsere; er sei unbewohnt. Aber schon Heraklit [ebenso wie Xenophon] erklärt den Mond für eine von Menschen bewohnte Erde ${ }^{159}$ ). Anaxagoras behauptet, der Mond sei ein von Menschen bewohnter Weltkörper, größer als der Peloponnes ${ }^{160}$ ). Ja, Lucien hat sogar Menschen auf dem Mond gesehen, und mit ihnen gesprochen ${ }^{159}$ ). Gilbert sagt, der Mond sei eine Erde, nur kleiner als die unsere; ebenso Henry Leroy und François Partice ${ }^{159}$ ). Nikolaus von Cusa ${ }^{161}$ ) und Giordano Bruno ${ }^{162}$ ) erklären sogar alle feurigen Weltkörper für bewohnt.

Der père Mersenne glaubte Wasser auf dem Mond, genau so wie auf der Erde zu sehen ${ }^{163}$ ). Descartes erklärt ${ }^{164}$ ): Quoique je n'infère point qu'il y ait des créatures intelligentes dans les étoiles ou ailleurs, je ne vois pas aussi, qu'il y ait aucune raison, pour laquelle on puisse prouver qu'il n'y en a point. Endlich auch Gassendi sagt, es gebe Gebirge auf dem Mond, viermal so hoch, wie der Olymp.

157) Desc. Med. III (I, 272).

${ }^{158)}$ Gass. Object. V (Cousin II, 140).

159) Cyr. Préface par Lebret: V-IX; angeführte Stellen: In dio Locré;

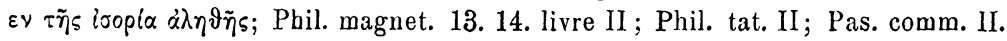

160) Kublenbeck: G. Brunos Dialoge. Einleitung III.

161) Cusa: De docta ignorantia II, 11.

162) Giord. Brunos Dialoge (Kuhlenbeck) 120 u. 143).

${ }^{163)}$ Cyr. Préface: Mersenne: Livre des questions inouyes cap. 9, 17.

164) Desc. Lettre à Chanut (IX, 50). 
Wir sehen, Cyrano hatte Vorgänger genug, die im Ernst behaupteten, was er zur scherzhaften Grundlage seines komischen, phantastischen Romans macht. Von seinen zahlreichen Nachfolgern bringt Brun eine vollständige Zusammenstellung ${ }^{165}$ ).

§ 29. Verwirklichung phantastischer Gedanken.

Zum Schluß möchte ich noch einige Proben des eigentümlichen Ahnungsvermögens anführen, das Cyrano vor allem auch auf physikalischem Gebiet bewies. Er erdenkt sich, um seine Leser zu vergnügen, Dinge, als bloße Ausgeburten seiner Phantasie, die wir heute teils in Wirklichkeit besitzen, teils mit größerem Ernst als durchführbar betrachten. So sind seine Flugmaschinen, mit denen er die Lüfte durchsaust, die Anfänge des Luftballons. Er ist der Erste, der ein merkwürdiges Instrument beschreibt, das wir heute besitzen, den Phonographen. Es sind die Bücher der Mondbewohner, die man ohne Benutzung der Augen lesen kann; denn es sind Kästen, in denen sich eine Art Uhrwerk befindet, mit unzähligen Rädchen und Nervchen und Rollen. Wenn man lesen will, so dreht man die Nadel auf das gewünschte Kapitel; und aus dem Apparat tönt es, wie eine menschliche Stimme ${ }^{166}$ ). Auch den Fallschirm wendet Cyrano schon an. Der Zufall lehrt ihn seinen Gebrauch. Bei einem unglücklichen Absturz von seiner Maschine hätte er leicht zu Schaden kommen können, wenn nicht sein weiter Mantel, in dem sich die Luft fing, sich so aufgebläht hätte, daß er ihn sicher und langsam zum Boden trug ${ }^{167}$ ).

$\S 30$. Resultat der Untersuchungen.

Was ist das Resultat unsrer Untersuchungen?

Wir haben Cyrano an seinen Platz in dem Rahmen der Entwicklung naturphilosophischer Ideen zu stellen versucht, und so vor allem sein Abhängigkeitsverhältnis von Descartes und Gassendi bestimmt.

165) Brun, 282-84; nebst Anm.

166) Cyr. I, 147.

${ }^{167)}$ Cyr. I, 34. 
Des ersteren gewaltiger Einfluß tritt uns gerade in Cyran. Gestalt klar entgegen. Obgleich Gassendis Schüler mufste er si doch den klaren Gedankengängen Descartes' unterwerfen, wie se ihn auch oft seine Neigung auf Gassendis Seite zurückzog. So j das ganze Fragment de Physique nichts als Wiederga Descartes'scher Gedanken. Sobald aber das Feld des Roma seiner Phantasie freien Spielraum läßt, gewinnt Gassendi nic selten wieder die Oberhand; und, vielleicht ihm selbst unbewuI legt er oft genug in die Produktionen seiner lebhaften Einbildunc kraft den Kern Gassendischer Lehren, deren Gegenteil er in de ernsteren Rahmen der gelehrten Abhandlung behauptet hatte. 